\title{
PRINCIPALES ARTRÓPODOS DE IMPORTANCIA MÉDICA. I. SCORPIONES, ARANEAE Y ACARI
}

\author{
Menandro S. Ortiz ${ }^{1}$
}

\section{SUMARIO}

Se realiza una presentación de los principales arácnidos de nuestro medio, para la comprensión e importancia que representan estos quelicerados para la salud humana, los que se comportan como parásitos u ocasionan daños de otra naturaleza, tal como ocurre con las arañas y escorpiones, otorgando datos sobre sus aspectos morfológicos, biológicos y de comportamiento.

Palabras claves: Chelicerata, parásitos.

\section{SUMMARY}

The following presentation discusses the main arachnids in our environment with the purpose of understanding and recognizing the importance that cheliceratas represent to human health, including those which behave like parasites (such as mites) or cause other types of damages (such as spiders and scorpions). Data on the arthropods morphological, biological and behavioural aspects are also discussed.

Keywords: Chelicerata, parasites.

\section{INTRODUCCIÓN}

Los artrópodos comprenden invertebrados cuyo cuerpo está dividido en tres regiones que pueden estar diferenciados o no. Cuando están claramente diferenciados, las regiones son cabeza, tórax y abdomen; sin embargo las dos primeras regiones pueden estar fusionadas constituyendo lo que se conoce como cefalotórax y abdomen. En el primer caso tenemos como ejemplo a los insectos y en el segundo caso se hallan por ejemplo las arañas. Un caso aparte lo constituyen los ácaros y las garrapatas, los que presentan una organización corporal muy particular.

$1 \quad$ Facultad de Medicina Humana y Facultad de Ciencias Biológicas. Universidad Ricardo Palma, e-mail: mortiz@urp.edu.pe 


\section{CLASE ARACHNIDA}

Los miembros de la Clase Arachnida son quelicerados terrestres, aunque pueden existir algunas especies acuáticas. Son en su mayor parte predadores, alimentándose principalmente de otros artrópodos. Presentan dos regiones corporales claramente distinguibles, el cefalotórax y el abdomen. No presentan antenas y las piezas bucales están conformadas por los quelíceros y los pedipalpos. Comprende varios órdenes, pero los que pertenecen a los escorpiones y arañas, usan veneno para inmovilizar a sus presas; son predadores. Parte de la digestión es externa. Muchos de ellos son nocturnos.

\section{ORDEN SCORPIONES}

Estos artrópodos tienen hábitos nocturnos y actúan con mucho sigilo. Son predadores. De día se ocultan bajo grietas, oquedades, rocas, piedras, etc., en ámbitos desérticos; aunque debe señalarse que existen especies que están relacionadas con la vegetación, viviendas humanas, incluso dentro de ellas.

Las especies que viven en los desiertos son más abundantes; que aquellas que viven en los bosques tropicales. La mayoría de ellos tienen una longitud que oscila entre los 3 a $9 \mathrm{~cm}$ de largo; aunque existen especies más pequeñas, o de lo contrario, pueden alcanzar mayores dimensiones.

El cuerpo de los alacranes consta de dos partes bien definidas: un cefalotórax o prosoma en cuyo aspecto medio dorsal se observan dos ojos simples, algo elevados y en los ángulos antero-laterales hay un pequeño grupo de dos a cinco ojos simples más pequeños. Los quelíceros ubicados en la parte anterior y central de esta región son trisegmentados. Consta de un segmento basal y dos segmentos distales, cuyos márgenes que se corresponden entre sí son dentados y les sirve para triturar a la presa. Cada uno de estos segmentos se denomina dígitus. Uno es fijo y el otro es móvil. Los pedipalpos, externos a los quelíceros, son bastante desarrollados y terminan en pinzas con los cuales capturan a las presas; usándolos también para el proceso de reproducción.

El abdomen subdividido en dos partes. La primera parte consta de siete segmentos anchos, ampliamente unido al cefalotórax; y la segunda parte presenta cinco segmentos delgados a manera de una cola y se denomina 
postabdomen. Consecuentemente son doce los segmentos abdominales. Le sigue una especie de ampolla que en buena cuenta viene a ser el aparato del aguijón, con el cual inyecta el veneno a la presa. Para ello levanta el postabdomen por encima del cuerpo, de modo que se arquea hacia delante para punzar e inyectar el veneno a la presa e inmovilizarla..

En la zona medio-ventral del primer segmento abdominal se halla el orificio genital, el cual está ocultado por un par de placas operculares. Posterior a estas placas genitales y fijado al segundo segmento abdominal se hallan los pectens (por su aspecto de un peine) basalmente fijados, lateralmente dirigidos y con los extremos distales libres. Son estructuras sensoriales que principalmente determinan la estructura del suelo.

Durante la etapa reproductiva el macho trata de encontrar a una hembra. Al encontrarla inicia un cortejo que puede tener variados aspectos de comportamiento. Seguidamente toma a la hembra con los pedipalpos y la estimula para que esta avance en varias direcciones por un determinado tiempo. Luego el macho deposita en el sustrato un espermatóforo que porta la cuota espermática. Entonces el macho se encarga de que esta estructura se ubique en el poro genital de la hembra, ingresando de esta manera la cuota seminal.

Todos los alacranes incuban los huevos dentro del gonoducto femenino, de modo que de las hembras emergen ejemplares vivos. El desarrollo tarda varios meses hasta aproximadamente un año. Al liberarse las crías, pequeñas aún, trepan inmediatamente al dorso de la madre, permaneciendo de ésta manera hasta que ocurre la primera muda, aspecto que sucede al promediar siete días. Posteriormente abandonan a la madre y empiezan una vida de manera independiente. Para alcanzar el estado adulto ocurren de cuatro a siete mudas, según la especie y tardan de seis meses a seis años alcanzar la madurez sexual. No presentan metamorfosis.

El veneno de la gran mayoría de alacranes es bastante tóxico para los invertebrados, no así para el ser humano; sin embargo existen especies que poseen venenos muy tóxicos, los que pueden ser mortales para el ser humano; como los que suceden con las especies que pertenecen a los géneros Androctonus y Centruroides. Hay venenos neurotóxicos muy dolorosos, los que provocan la parálisis de los músculos respiratorios y hasta paro cardíaco. 
Cuando ocurre una picadura, las manifestaciones clínicas locales son dolor, edema, eritema e infiltración subcutánea que puede terminar en la configuración de una placa violácea, la cual desaparece al cabo de cuatro a seis días. A veces puede haber fiebre, prurito y fenómenos secretorios como la rinorrea. En casos severos se asocian espasmos musculares, convulsiones y otros síntomas similares al latrodectismo.

En algunas ocasiones, cuando la evolución es grave, existe acción central neurotóxica del veneno, el que actúa como un potente estimulador del sistema nervioso simpático, se produce hipertensión arterial, edema pulmonar y falla circulatoria periférica, entrando el paciente en estado crítico, pudiendo llegar a un desenlace fatal en pocas horas.

La letalidad del escorpionismo, nombre con el que se conoce los accidentes que ocurren con este grupo de artrópodos, es relativamente baja, dependiendo del agente ponzoñoso y de las características y condición general del individuo picado. La causa de la muerte es, en la mayoría de los casos, parálisis respiratoria por compromiso bulbar.

\section{Hadruroides lunatus (Koch, 1867)}

Es la especie que habita en nuestro medio. Esta especie, que pertenece a la familia Vejovidae, es conocida como el "alacrán de los pedregales" y se halla ampliamente distribuida en la vertiente occidental de los Andes, desde el sur de Colombia hasta el norte de Chile, incluyendo las islas Galápagos. En el Perú está distribuido principalmente a lo largo de la costa, penetrando en la región de la sierra, sin llegar a trasponer la barrera constituida por la Cadena de los Andes, Tiene como hábitat preferido los lugares pedregosos. La alimentación de esta especie está basada en pequeños insectos, como coleópteros, crustáceos del género Porcellio y arácnidos que habitan en diversos lugares. Para alimentarse, el alacrán coge a la presa fuertemente con los pedipalpos, los que tienen formas de pinzas, inyectándole inmediatamente el veneno por medio del aguijón, localizado en la parte caudal del abdomen. El veneno de éste alacrán posee fracciones tóxicas con actividad paralizante sobre las presas de pequeño tamaño. Esta actividad tóxica es producida por la acción de las neurotoxinas polipeptídicas. La electroforesis en acetato de celulosa ha permitido la tipificación de hasta 12 grupos proteicos en el veneno de Hadruroides lunatus. 
Respecto a los accidentes que ocurren con los seres humanos, ha sido reportado que estos son leves, sintiéndose una cierta elevación de la temperatura en el lugar donde ha penetrado el aguijón, acompañado con edema y dolor, el que va disminuyendo después de algunas horas. En los casos graves existe excitación y dolor. La acción neurotóxica se dirige por las terminaciones nerviosas, con presencia de vasoconstricción periférica que puede durar entre dos a seis horas. La acción de los alacranes inoculando el veneno sobre humanos, se denomina escorpionismo.

Sin embargo existe autores que han trabajado con éste veneno, identificando y denominándola a la toxina HI3, la que actúa sobre músculos esqueléticos. Se caracteriza por producir contracción muscular y parálisis. Escobar et al. (2003) han determinado que esta toxina inoculada en el músculo gastrocnemius produce liberación en el plasma de creatina kinasa (CK) y lactato deshidrogenasa (LDH). Provoca también la liberación de otras proteínas musculares. Además HI3 es capaz de incrementar los niveles de calcio intramuscular. Este último efecto, al parecer es el más usual, afectando la permeabilidad del sarcolema y provocando la fuga de proteínas musculares. Además el incremento de los niveles de calcio podría generar hipercontracción y destrucción de fibras musculares, por aumento de las proteasas dependientes de éste ión. Incluso puede llegar a producir necrosis muscular.

\section{ORDEN ARANEAE}

Comprende a artrópodos comúnmente conocidas como arañas. Sin embargo debe aclararse que la mayoría de ellas pertenecen al Suborden Labidognatha, llegando a medir desde los $0.5 \mathrm{~mm}$ hasta aproximadamente $4 \mathrm{~cm}$, incluyendo la envergadura de las patas. Aquellas que son más grandes y peludas son las llamadas migalas y pertenecen al suborden Orthognatha. Existen especies de hábitos diurnos y nocturnos. Todas son predadoras. Todas elaboran hilos de seda; pero el uso que le otorgan es variado. Por lo general la usan para tejer redes para atrapar a la presa, otras especies las usan para colgarse luego de atrapar a la presa o para desplazarse y otras para cubrir las paredes de las grietas en donde viven.

Presentan el cuerpo dividido en dos partes claramente diferenciadas: el cefalotórax o prosoma y el abdomen u opisthosoma. Estas dos regiones están unidas por un corto y delgado pedicelo. En la parte anterior 
del cefalotórax existe dos pares de apéndices; el par interno son los quelíceros, cada uno de ellos conformados por dos segmentos, uno basal, usualmente cilíndrico y uno distal con aspecto de garra. Justamente, la implantación del segmento basal sirve para diferenciar a los subórdenes. Aquellos que pertenecen al Suborden Labidognatha presenta la base del quelícero implantados verticalmente en la parte anterior del cefalotórax; y el segmento con aspecto de garra se orienta hacia la parte interna. Consecuentemente, el movimiento de ellos es lateral para inyectar el veneno. En el caso de aquellos Araneae que pertenecen al suborden Orthognatha presentan el segmento basal del quelícero continuando el eje del cuerpo o ligeramente dirigido hacia abajo; y la orientación del segmento con forma de garra, cuando están en reposo es ventral al primero. La aplicación de ellos para inyectar el veneno es de arriba hacia abajo.

Los apéndices anteriores y externos del cefalotórax son los pedipalpos, estructuras sensoriales, que incluso sirven para determinar los sexos. La parte distal del macho presenta una dilatación característica para cada especie, llamado bulbo copulatorio, no así el de la hembra.

En la parte antero-dorsal del cefalotórax presenta generalmente ocho ojos simples, cuya forma, tamaño y disposición tienen importancia para determinar fundamentalmente a los grupos sistemáticos a los que pertenecen las arañas. También existen arañas con solo seis ojos, como por ejemplo las especies que pertenecen al género Loxosceles.

El abdomen, está conformado por segmentos, pero usualmente estos no son notorios. Antero-ventralmente hay un surco transversal denominado surco epigástrico. Los poros genitales, del macho y hembra, se hallan en la mitad del surco y los espiráculos de los libros pulmonares están a los lados de éste. La parte ventro-caudal del abdomen presenta los spinnerets o hileras, estructuras que sirven para que salgan los hilos de seda, los que provienen de una glándula ubicada al interior del abdomen.

La así llamada cópula (por no existir el phallus), consiste en la introducción del bulbo copulatorio en el poro genital de la hembra. Los espermatozoides se almacenan en los receptáculos seminales o espermatecas. Para este acto existen diversas formas de cortejo. Fundamentalmente, la araña hembra debe identificar al macho como pareja y no como alimento. Para ello entran a tallar una serie de complejos mecanismos sensoriales físicos y químicos. 
La hembra deposita los huevos en una ovisaco, construido en base a hilos de seda. Después de la incubación emergen las formas juveniles, dispersándose inmediatamente. No presentan metamorfosis.

\section{Loxosceles laeta (Nicolet, 1849)}

Esta especie pertenece al suborden Labidognatha, debido a que el segmento basal de cada quelícero se implanta verticalmente en la parte anterior del cefalotórax o prosoma. Es de color parduzco, siendo algo más oscuro en el abdomen y el cuerpo está cubierto por una pilosidad corta y abundante. Mide un promedio de $4 \mathrm{~cm}$ de longitud, incluido la envergadura de las patas. El cefalotórax presente en el aspecto dorso anterior solo tres pares de ojos simples, dispuestos un par anterior y dos pares laterales, formando un triángulo.

No presentan metamorfosis. Su desarrollo es a través de mudas hasta alcanzar el estado adulto. La hembra deposita alrededor de 200 huevos dispuesto en un ovisaco, elaborados con hilos de seda. Este receptáculo es redondeado y se ubica en la vecindad de la tela, la que es irregular. Las arañas que emergen primero, ingieren a las restantes. Después de algunas semanas salen del ovisaco y se dispersan por las proximidades, donde establecen nuevos nidos. El ciclo biológico dura aproximadamente un año.

La picadura accidental de ésta especie al ser humano se conoce con el nombre de loxoscelismo. Se trata de una especie de hábitos solitarios, ubicándose en el interior de las casas, de allí que algunas personas la conozcan como "araña casera". Usualmente se halla en los rincones altos y sombríos, por ello también se le conoce como la "araña de los rincones". También puede hallársele detrás de cuadros y muebles. En estos lugares teje una tela laxa, algodonosa y sucia, que le sirve de refugio durante el día y hacía donde arrastra a las presas que captura. Su actividad es principalmente nocturna; y accidentalmente puede caminar sobre las partes expuestas de una persona (brazos, piernas) cuando ésta duerme; y que inconscientemente al tratar de retirarla por la molestia que le causa, la araña para defenderse introduce sus quelíceros e inyecta el veneno. Otra forma accidental es cuando la víctima se pone la ropa o zapatos, estando la araña oculta en éstas indumentarias. 
El veneno de ésta especie está formado por los siguientes componentes proteínicos; la hialurodinasa (factor de difusión), la desoxirribonucleasa, la ribonucleasa, la lipasa, la fosfatasa alcalina y la esfingomielinasa-D, fracción esta última responsable de la citotoxicidad del veneno.

El veneno de Loxosceles laeta actúa de dos maneras: la primera de ellas y con mayor prevalencia es la necrotizante. Aquí hay dolor intenso, en donde posteriormente aparece una mancha violácea, denominada placa livedoide. Ella indica el inicio de la dermonecrosis. La úlcera que aparece es de difícil cicatrización a menos que se aplique el suero antiloxoscélico.

El segundo tipo de acción del veneno es cuando alcanza el torrente sanguíneo, es decir se hace sistémica y ataca a los glóbulos rojos destruyéndolos. La esfingomielinasa-D es la fracción responsable de la citotoxicidad, ocasionando la hemólisis, llegando a la insuficiencia renal y posteriormente la muerte. La prevalencia de éste tipo de acción del veneno de ésta araña es menor.

\section{Latrodectus mactans Fabricius, 1775}

Se trata de una especie que pertenece al mismo suborden que Loxosceles laeta. Presenta dimorfismo sexual, es decir, la hembra y el macho son de tamaño y color diferentes. Así, la hembra que mide un promedio de $4 \mathrm{~cm}$ de longitud, incluyendo las patas, presenta una coloración negra, mostrando en la parte ventral del abdomen un par de triángulos opuestos de color rojo.

El cefalotórax presenta en el aspecto ánterodorsal ocho ojos simples, dispuesto en dos hileras paralelas conformadas por cuatro ojos cada una de las hileras. El abdomen de la hembra es relativamente grande y globoso. El macho es significativamente más pequeño y por lo general de color grisáceo, el que después que fecunda a la hembra es usualmente devorado por ésta, razón del apelativo en nuestro medio de "viuda negra".

No presenta metamorfosis. Su desarrollo simplemente lo efectúa a través de mudas, hasta llegar al estado adulto. La hembra deposita un rango de huevos que oscila entre 100 a 500, encerrados al interior de un ovisaco, el que es esférico e impermeable con un centímetro de diámetro, de color blanquecino a parduzco. Al cabo de tres semanas emergen los juveniles, los que se pigmentan paulatinamente durante los ocho meses en que 
permanecen dentro del ovisaco. Presentan canibalismo. Usualmente sus presas son insectos que capturan por medio de su tela estratégicamente ubicada. Son de hábitos diurnos y viven en zonas rurales, nunca en el interior de las casas.

Tienen actividad diurna y el contacto con la persona es accidental, atacando preferentemente los brazos y las piernas. El ataque accidental de ésta araña se conoce como latrodectismo.

El veneno de ésta especie contiene al menos seis componentes de naturaleza proteínica, siendo la más importante la alfa-latrotoxina, la que afecta la sinapsis neuromusculares con liberación de acetilcolina y norepinefrina, neurotransmisores que estimulan excesivamente la placa neuromotora.

La acción del veneno es neurotóxica y se concentra en el sistema nervioso vegetativo. La picadura produce inicialmente una sensación de quemazón, luego el dolor migra hacia las partes iniciales de los brazos y/o piernas. Existen contracciones musculares y dificultad para los movimientos. Cuando el dolor asciende al tórax hay dificultad para respirar y se produce una sensación de muerte inminente. Acompañando a todo este cuadro, existe también sudoración, secreción lagrimal, contracción de esfínteres, aumento de la presión arterial y priapismo.

\section{CLASE ACARI}

Los miembros que pertenecen a la Clase Acari se les conocen comúnmente como ácaros y garrapatas. Los ácaros son diminutos o microscópicos y las garrapatas son macroscópicas. Los primeros tienen un comportamiento alimenticio variado, según las especies; así hay los que se alimentan de linfa, de sangre, los hay fitófagos y predadores. En cambio todas las garrapatas son hematófagas. Entre estos dos grandes grupos existen especies que representan un problema directo, relacionado con la salud del ser humano, como por ejemplo son los hematófagos, los que a la vez se pueden comportar como vectores de agentes etiológicos (virus, bacterias) de diversas enfermedades.

Presentan el cuerpo dividido en dos partes claramente diferenciables: el gnathosoma y el idiosoma. La primera parte presenta los cuatro apéndices señalados para el caso de los Araneae y Scorpiones, por ser quelicerados. 
Así, están los quelíceros trisegmentados, los que pueden variar en forma dependiendo del hábito alimenticio que presenten. Si se alimentan de linfa o sangre, presentan los dígitos (los dos segmentos distales, el dorsal es rígido y el ventral es movible) algo alargados y terminados en punta. En cambio si son predadores, los dígitos tienen función trituradora, teniendo para ello los márgenes que se corresponden entre sí, dentados. En el caso de las garrapatas, ventralmente a los quelíceros se halla el hipostoma, una placa con dientes agudos, dirigidos hacia la parte posterior, los que les permite anclarse en la piel del hospedero. Externamente a los quelíceros están los pedipalpos formados por seis segmentos articulados.

En el idiosoma se presentan en la parte anterior cuatro pares de patas, cada uno de ellos con siete segmentos. Justamente la parte del idiosoma en donde hallan las patas, diversos autores la denominan podosoma; y la parte posterior, sin apéndices locomotores, se denomina opisthosoma.

La inseminación es directa en casi todos los Acari y ocurre por medio del phallus; sin embargo existen también inseminación por medio de espermatóforos, cogiéndolo la hembra sin ayuda del macho. Existen también otras formas de inseminación.

Todos los Acari son ovíparos, aún cuando en algunos casos los huevos puedan incubarse dentro del cuerpo de la madre; y más aun, puedan completar su desarrollo para salir al exterior de la madre en estado adulto.

Los huevos al eclosionar dan origen a una forma inmadura, la que se caracteriza por tener solamente tres pares de patas, la que recibe el nombre de larva. Posteriormente suceden varias mudas, las que llegan a tener cuatro pares de patas, denominándose en este caso ninfas, para finalmente dar lugar a las formas adultas, sexualmente maduras. Con cierta frecuencia presentan partenogénesis; y esta puede ser arrenotokia cuando todos los descendientes son machos, o puede ser también telitokia, cuando todos los descendientes son hembras.

\section{Sarcoptes scabiei De Geer 1778}

Se trata de una especie de ácaro que ataca la piel del ser humano, la epidermis, ocasionando el mal conocido como escabiosis. Por tal motivo se le conoce comúnmente como el "arador de la sarna". Esta especie presenta una serie de variedades que atacan de manera similar a diferentes 
hospederos; sin embargo la que ectoparasita al ser humano es Sarcoptes scabiei var. hominis. Es un ectoparásito cosmopolita, es decir se le halla en todo el mundo. Es muy contagiosa, de compromiso grupal y exclusivo del ser humano. Ello quiere decir que es fácilmente transmisible por el contacto directo o través de fomites (prendas de vestir, sábanas, toallas, etc.), teniendo en cuenta fundamentalmente el nivel socio-económico y cultural de la población, la época del año, las características geográficas y la ocurrencia de catástrofes naturales.

El macho que mide de 150 a $250 \mu$, poco después de fecundar a la hembra $(300-450 \mu)$ muere, siendo esta la que consecuentemente presenta una mayor longevidad, con la finalidad de depositar los huevos para que emerja una próxima generación; y consecuentemente es la que afecta mayormente la piel del ser humano, es decir es el principal ectoparásito, la que labra la parte externa de la piel siempre hacía adelante, dado que presenta en el dorso del idiosoma una serie de setas largas que le impiden retroceder. A la acción de la producción del túnel, conlleva un intenso prurito; sobre todo en horas nocturnas. Es en el túnel, después de fecundada, donde deposita los huevos y al cabo de dos a tres días emergen las larvas, las que sólo tienen tres pares de patas. Posteriormente de tres a cuatro días, después de la correspondiente muda, se transforma en ninfa, reconociéndose esta etapa porque presenta cuatro pares de patas. Finalmente llega al estado adulto sexualmente maduro. La hembra después de la digestión excreta catabolitos que producen una reacción alérgica, lo que causa escozor. El prurito se torna en una manifestación importante porque lleva al rascado y este a la vez a una infección (piodermitis) que enmascara las verdaderas lesiones. Estas lesiones se hallan principalmente en las muñecas, los aspectos laterales de los dedos, las manos, los codos y las nalgas. En el caso del varón puede invadir el prepucio y el glande y en el caso de la mujer las areolas de los senos.

La escabiosis por lo general no está presente en las personas que cultivan buenos hábitos higiénicos, no obstante, puede haber también contagio.

El diganóstico se realiza mediante la búsqueda de parásitos adultos en las lesiones. El tratamiento de la escabiosis no sólo debe hacerse a la persona con dicho mal, sino a todo el grupo humano que constantemente están en contacto con la persona infestada. 


\section{Demodex folliculorum Simon, 1842}

Se trata de una especie de ácaro microscópico de la Familia Demodicidae y cuyo parasitismo se conoce como demodicidosis. Tiene un aspecto alargado, vermiforme con ectoesqueleto finamente estriado a la altura del idiosoma. Sus estados de desarrollo son huevo, larva, ninfa y adulto.

Vive en los folículos pilosos de la cara, pestañas, cejas y barba. Principalmente se ubican en la cara, alrededor de la nariz y de los ojos en pequeña cantidad (no más de cinco en una muestra de pápula que se examine); sin embargo, excepcionalmente un aumento exagerado en número puede provocar cuadros inflamatorios más severos y crónicos. Este ácaro puede también agravar los cuadros de acné, sobre todo al acné rosácea. A menudo se le conoce como el "ácaro del folículo".

$\mathrm{Su}$ presencia también ha sido relacionada con diversas enfermedades oftalmológicas como conjuntivitis, blefaritis eccematosa crónica, chalazón e intolerancia al uso de lentes de contacto. Los signos más frecuentes en relación a la blefaritis son el prurito, la caída de pestañas y el edema palpebral.

Referido a su estructura corporal, provoca una reacción granulomatosa a cuerpo extraño, lo que produce un bloqueo mecánico que puede explicar la aparición de blefaritis y chalazón. El acumulo de costras en la base de las pestañas, corresponde a excrementos, exudados del folículo o proliferación epitelial.

Una serie de estudios han demostrado que estos ectoparásitos y sus productos estimulan mecanismos de hipersensibilidad con producción de anticuerpos.

\section{Ornithonyssus sylviarum (G. Canestrini \& Fanzago, 1877)}

Durante los inicios del año 2011 se aprecia un incremento de una zoonosis emergente relacionada a ácaros de palomas, caracterizada por un cuadro clínico polimorfo, con presencia de lesiones papulares cupuliformes, eritematosas y excoriaciones por el rascado debido al intenso prurito, por la presencia de ésta especie reportada por Téllez et al. (2008), autores de quienes se ha tomado segmentos importantes de su trabajo, en razón de la experiencia obtenida y por ser el primer reporte hallado en nuestro país. La picadura de estos ácaros pueden confundirse con las provocadas 
por otros insectos, pero predomina en ellas una mayor reactividad de las lesiones cutáneas. Todos los ácaros pueden provocar prurito o reacciones alérgicas debido a las proteínas salivales depositadas durante la succión de sangre.

En las áreas metropolitanas extensas, especialmente en las que las palomas tienden a reunirse, es frecuente verlas descansar en las ventanas, azoteas, tragaluces o en los jardines de las casas o parques; por ello el ácaro de la paloma puede llegar al ser humano, infestarlo y producirle una dermatitis papular.

En la ciudad de Lima se ha incrementado la población de palomas la que es ahora considerada una plaga, ensuciando con sus deyecciones y alimentándose de los desechos de basura dejados por las personas.

Ornithonyssus sylviarum pertenece a la Familia Macronyssidae; es de color blanco translúcido hasta que toman sangre, con lo cual se tornan de color rojizo. Puede subsistir por varias semanas; es un ectoparásito permanente que pone sus huevos, desarrolla y pasa la mayor parte de su vida sobre su hospedador. Existen reportes que pueden transmitir diversos agentes etiológicos de enfermedades a las aves. Esta especie de ácaro habita usualmente en América del Norte, Australia y Nueva Zelanda.

En las personas afectadas, hospedadores accidentales, se observan múltiples lesiones polimórficas con pápulas cupuliformes con base eritematosa, fundamentalmente en el cuello, brazos, tórax, abdomen y piernas. Además ocasiona indirectamente excoriaciones debido al rascado ocasionado por el intenso prurito presente a cualquier hora del día. Lo que se debe agregar a lo señalado es que existe en todos los patrones un infiltrado inflamatorio en la dermis. Las personas que se ven afectadas, de alguna manera tienen como antecedente estar cercana a las palomas o a sus nidos.

La irritación producida por la picadura y aumentada por la saliva que el ácaro introduce, puede causar una reacción local debido a la histamina que origina las pápulas típicas de éste cuadro. 


\section{REFERENCIAS BIBLIOGRÁFICAS}

Arndt, K.A., Bowers K,E. Chuttani A.R., 1995. Manual of dermatologic therapeutics. Little, Brown and Company Boston: 120-127.

Burns D.A. The treatment of human ectoparasite infection. Br. J. Dermatol. 1991, 125: 89-93.

Cáceres, I.E., P.G. Aguilar y O. Meneses.1972. Escorpiones y Escorpionismo en el Perú.- II. Efectos del Veneno del "Escorpíón de los Pedregales" en la Costa Central. Rev. Per. Ent. 15 (1): $38-43$.

Castro, G. A. Zavaleta y R. Castro de la Mata. 1981. Variación Estacional del Veneno de Hadruroides lunatus (Koch) y su Actividad Paralizanate sobre Porcellio laevis Koch (Crustacea: Isopoda). Rev. Per. Ent. 24 (1): $71-74$.

Chosidow O. 2000. Scabies and pediculosis. Lancet, 355: 819-826.

Crawford G.H., Pelle M.T., James W.D. 2004. Rosacea: I. Etiology, pathogenesis and subtype classification. J. Acad, Dermatol. 51 (3) 327-341.

Escobar, E., C, Rivera y L. Tincopa. 2003. Acción de la toxina HI3 sobre músculo esquelético. Rev. Per. Biol, 10 (1): 88 - 92

Escobar. E, L. Flores, C. Rivera. 2008- Péptidos antibacterianos de los venenos de Hadruroides mauryi y Centruroides margaritatus. Rev. Per. Biol. 15 (2)

Lacey N., Kavanagh K. Tseng S.C. 2009. Under the lash: Demodex mites in human diseases. Biochem. (Lond.). 31 (4): 2-6

Miller T. 1992. Latrodectism: bite of the black widow spider. American Family Physian, 45: 181-187.

Náquira C., R. Verano, M. Ortiz, 1999. Manual de Parasitología. Facultad de Medicina Humana, Universidad Ricardo Palma. 200 pp.

O’ Donnell B.F., O' Loughlin S., Powell F.G. 1990. Management of crusted scabies. Int. J. Dermatol, 29: 258 - 266-

Parra, D-, M. Torres, J. Morillas, P. Espinoza. 2002. Loxosceles laeta, identificación y una mirada bajo microscopía de barrido. Parasitol. Latinoam. 57: 75-78. 
Scott, S.; Craig, T. 2000. Pest of Paradise: First Aid and Medical Treatment of Injuries from Hawaii"s Animals. Univ. Hawaii Press (Honolulu) 190 pp.

Sedeño Cruz, I., E. Novoa, V. Padrón, F. García y R. San Martín. 2006. Blefaritis por Demodex folliculorum. Diagnóstico y tratamiento. Rev. Cubana Oftalmol., 19 (1):

Téllez, M.L., C. Sordo, A, Ruiz, S. Tucto y A. Manrique. 2008. Dermatosis por ácaros de palomas. Primer reporte de la presencia de Ornithonyssus sylviarum en el Perú. Folia dermatol. Perú, 19 (2): $63-68$.

Zavaleta, A., E. Bustamante y R. Castro de la Mata. 1979. Escorpiones y Escorpionismo en el Perú.- VIII: Acción del Veneno de Hadruroides lunatus sobre el Intestino Aislado de Rata. Rev. Per. Ent. 22(1): $75-81$. 